\title{
The Role of TRPV1 Receptors in Vasoregulation
}

\author{
Róbert Pórszász* \\ Department of Pharmacology and Pharmacotherapy, University of Debrecen, Hungary
}

*Corresponding author: Róbert Pórszász, Department of Pharmacology and Pharmacotherapy, University of Debrecen, Nagyerdei krt. 98. H4032 Debrecen, Hungary.

To Cite This Article: Róbert Pórszász. The Role of TRPV1 Receptors in Vasoregulation. 2020 - 10(2). AJBSR.MS.ID.001489. DOI: 10.34297/ AJBSR.2020.10.001489.

Received: 眥 August 06, 2020; Published: 眥 September 03, 2020

\begin{abstract}
Transient Receptor Potential V1 (TRPV1) receptor is under investigation for several decades to develop a sensory neuron specific analgesic substance, but in contrary of the intense effort success is awaiting. A pile of experimental results gathered about the non-neural location of TRPV1 receptor in the last two decades. The physiological role of these receptor locations is not well understood and the pharmacological exploitation is not elaborated. The present review focuses on the role of TRPV1 receptors in vasoregulation and blood pressure homeostasis including Bezold-Jarisch reflex, baroreflex sensitivity, sensory neuron dependent vasodilation, endothelial cell mediated vasomotion, arteriolar Smooth Muscle Cell (SMC) related vasoconstriction and visualization of TRPV1 receptors on SMCs by reporter mice.
\end{abstract}

Keywords: TRP channels, TRPV1, Vasoregulation, Capsaicin, Baroreflex, Bezold-Jarisch Reflex, Vasoconstriction

\section{Introduction}

The Transient Receptor Potential Vanilloid 1 receptor (TRPV1) formerly referred to as the Vanilloid Receptor 1 (VR1) or the Capsaicin Receptor, plays an important role in pain sensation and can be activated by acidic $\mathrm{pH}$ (protons), noxious heat (over $43^{\circ} \mathrm{C}$ ) and by certain endogenous (anandamide, 12-S-HPETE etc.) and by exogenous substances such as capsaicin, resiniferatoxin (RTX), piperine and other chemicals [1], for details see (https://www.guidetopharmacology.org/GRAC/ FamilyDisplayForward?familyId=78). It is valid for TRPV1 channels that they build up from the following from $\mathrm{N}$ to C termini: N TerminusAnkyrin Repeat Domain (ARD) with 6 ankyrin repeats-linker domain-S1-S4 transmembrane domains (TMD)-S4-S5 linker-S5-S6 pore forming TMDs ( $\mathrm{S} 6 \pi$-helix)-amphipatic TRP helix regions-C terminus. Both $\mathrm{N}$ and $\mathrm{C}$ termini are located intracellularly and the receptor assembles as tetrameric/homotetrameric complexes [2]. The basis of these discoveries was laid down in the lab of David Julius by the receptor cloning around two decades ago [3] and it boosted the research to clarify the role of the TRPV1 receptor in sensory neuron functions summarized in excellent reviews [4-6].

Culinary usage of chillies and peppers (Capsicum fruits) originates from ancient civilizations as food additives. The pungency rate is measured by the subjective Scoville organoleptic test (Scoville Heat Unit, SHU), thus Resiniferatoxin (RTX) proved to be the strongest with $16 \times 10^{9}$, but capsaicin 1000 times weaker and represents $16 \times 10^{6}$ SHU but pungency determined by the eye wiping test showed the opposite order, only around $60 \%$ of eye wipes produced by RTX than that of capsaicin [7]. The capsaicin containing Hungarian "paprika" pungency level is around 5002,500 SHU, which is relatively "weak" compared to the police grade lachrymatory pepper spray $(5,300,000 \mathrm{SHU})$. Nowadays, the capsaicin content of various spices can be determined objectively with high performance liquid chromatography [8]. Capsaicin dissolves very well in ethanol, but milk drinking independently of its fat content is the best choice to mitigate burn caused by capsaicin containing spice [9].

It was believed for a long time that TRPV1 receptors expressed only on neurons (especially on primary sensory neurons) and participate in nociception and pain sensation $[4,5]$. The various extra neuronal expressions started to be studied only in the past two decades. TRPV1 was described in hypothalamic nuclei involved in the control of energy homeostasis [10], several human skin cell populations (e.g. keratinocytes) [11], vascular endothelial cells [12], vascular smooth muscle cells [13,14], immune cells [15], hepatocytes [16] and human platelets [17]. Here should be stressed, that TRPV1 mapping was based on visualization of TRPV1 receptors 
with antibodies generated against it, but Tóth et al. [18] reported that several commercially available rat anti-TRPV1 antibodies were not selective for TRPV1. Moreover, the visualization of TRPV1 expressed in smooth muscle cells or sensory neurons depends on the source of the commercially available antibody [18].

The same was found by Sand et al. [19] in mice: anti-TRPV1 antibodies lacked specificity for TRPV1 and they could not find functional TRPV1 expression in murine isolated endothelial or smooth muscle cells. Thus, the use of capsaicin as a functional control on intracellular $\mathrm{Ca}^{2+}$ elevation has a great importance. The possible up and/or downregulation of TRPV1 receptors according to certain pathological conditions is conceivable as well and the presence of vanilloid-insensitive TRPV1 splice variants can make more complex the picture [20]. Other methods, like measurement of specific mRNA level or the use of TRPV1 KO mice can help to clarify the role of TRPV1 on non-neural cells. The classical whole living conscious and anaesthetized animal experiment results become more important in decision about the relevance. So, the TRPV1 expression in non-neuronal locations will be reconsidered and this will be supported by new techniques, like the use of Reporter Mice (a dedicated section in this review).

The aim of the present review is to give a brief overview of the possible role of TRPV1 receptors in vasoregulation and possibly blood pressure regulation under physiological and pathophysiological conditions. Several drug groups and substances exist in the management of high blood pressure, but the so-called essential hypertension is not curable and the patients should take the antihypertensive treatment for lifelong owing to, inter alia, our insufficient knowledge about the mechanisms involved in the initiation of high blood pressure. The pharmacology and the pharmacological exploitation of TRPV1 receptors might let us closer to the proper solution. Considering this goal, TRPV1 receptors can have function in cardiovascular reflexes, i.e. Bezold-Jarisch reflex, baroreflex and can produce influence directly on vessels targeting smooth muscle cells, endothelial cells and even the innervating neurons. In the following sections these fields will be touched.

\section{Capsaicin-Induced Bezold-Jarisch Reflex and Accompanying Haemodynamic Changes}

Several decades ago, experiments were performed to clarify the hemodynamic effects of intravenously administered capsaicin. Early findings from the 50s and 60s of the past centuries described in detail the effect of capsaicin on blood pressure, heart rate and respiration when it was administered intravenously to dogs, cats and rats. The triad of hypotension, bradycardia and apnea (Bezold-Jarisch reflex, pulmonary chemoreflex) was evoked after intravenous bolus injections of ascending doses of capsaicin and no desensitization to it was described [21]. Bolus injection is required, because infusion of capsaicin is unable to evoke the reflex.
Perineural application of capsaicin to the vagal cervical nerves in cats blocked or highly reduced the triad evoked by intravenous bolus capsaicin administration, but had no effect on the responses evoked by phenybiguanide and veratrine, thus the authors concluded that chemo-specifically different populations of vagal afferent fibres are responsible for the reflex mediation [22].

ThecommonTRPV(V1,V2,V3)activator,2-aminoethoxydiphenyl borate (2APB) has been shown to evoke the reflex as well [23]. The effect of $3 \mathrm{mg} / \mathrm{kg} 2 \mathrm{APB}$ was comparable to that of capsaicin administered at a dose of $1 \mu \mathrm{g} / \mathrm{kg}$. The $2 \mathrm{APB}$ evoked chemoreflex was abolished by ruthenium red and attenuated by capsazepine, indicating the participation of not only TRPV1 receptors but other receptorial actions of 2APB. Perineural capsaicin treatment of both vagal nerves (known to defunctionalize TRPV1 expressing neurons) blocked 2APB and capsaicin evoked pulmonary chemoreflex and even single-fibre recordings proved the individual pulmonary $\mathrm{C}$ fibre activation by $2 \mathrm{APB}$.

The receptorial site of the reflex arch locates in the inferoposterior wall of the left heart ventricle and in the lungs. The vagal nerve serves for both afferent and efferent part of the reflex. The reflex induced bradycardia and hypotension (which is considered as Phase I part of the response obtained with capsaicin administration) generally followed by an increase in blood pressure (Phase II part). These reactions can be evoked by anandamide [24] and methanandamide with the difference that both anandamides elicited a more prolonged decrease in blood pressure (Phase III), but capsaicin was free of this effect. The rank of potency in eliciting the Phase I part is capsaicin > methanandamide > anandamide [21]. Interestingly, after the evoked Bezold-Jarisch reflex (Phase I) capsaicin caused blood pressure increment (Phase II) which remained after the administration of atropine, ganglion blocking Tetraethylammonium (TEA), or after the spinalization of the animal [25], thus it was attributed to direct vascular smooth muscle cell excitation by capsaicin leading to vasoconstriction.

The TRPV1 receptor antagonists capsazepine and ruthenium red were able to block only the Phase I reflex mediated part of the blood pressure change [21]. No blocking effect was observed on the Phase II brief vasopressor effect. A CB1 receptor antagonist, SR 141716 could block only the Phase III reactions, but Phase I and Phase II remained intact suggesting that CB1 receptors play a role only in the prolonged blood pressure reduction, but neither in the Bezold-Jarish reflex nor in the brief vasopressor effect [21], the mechanism of which still remained unknown. Even the nonpsychoactive substance of marihuana, Cannabidiol (CBD) could evoke Bezold-Jarisch reflex with the help of TRPV1 receptors since this effect was capsazepine (one selected dose of $0.4 \mathrm{mg} / \mathrm{kg}$ ) sensitive in around 60\% [26]. CBD showed instantly developing tachyphylaxis even after a single dose but it was unable to modify 
the capsaicin-induced Bezold-Jarisch reflex and could not evoke a long-term functional unresponsiveness (with a not proper word: desensitization) [26].

On the contrary, former experiments were unable to prove the effectiveness of CBD on pulmonary chemoreflex [27]. This controversy might originate from the usage of different anaesthesia (urethane vs. pentobarbital), from different source of CBD ( $(-)$-cannabidiol (THC Pharm GmbH, Frankfurt, Germany for in vivo and Tocris Cookson, Bristol, UK for in vitro studies vs. Sigma 4 Aldrich) and from different doses of administered CBD $(3,10,20 \mathrm{mg} / \mathrm{kg}$ vs. maximum cca. $6 \mathrm{mg} / \mathrm{kg}(2000 \mu \mathrm{g} / \mathrm{rat})$ with a very low sample number $(\mathrm{n}=1$ !) $)$. Electrophysiological recording of vagal pulmonary $\mathrm{C}$-fibre single units in anaesthetized mice revealed the modulatory effect of Tumor Necrosis Factor Alpha (TNF $\alpha$ ) on capsaicin induced TRPV1 activation. TNF $\alpha$ enhanced significantly the reflex (hypotension and bradycardia), serving the basis of altered pulmonary reflexes in inflammatory states of the lung [28]. TNF $\alpha$ can act on epithelial cells in promoting the transmigration of leukocytes via a TNFR1-dependent signalling pathway [29].

To analyse the nature of blood pressure increase after capsaicin administration, a hind-limb auto perfusion technique was developed [13]. Earlier, several publications from the laboratory of Eric Colquhoun proved that intraarterial capsaicin administration into the perfused hind-limb of the rat using artificial solution with the help of perfusion buffer reservoir and oxygenation by silastic lung evoked perfusion pressure increase, but differences were observed at low or high dose of capsaicin in oxygen consumption [30-32] They concluded that different types of receptors might exist (VN1 and VN2), but both presumed receptors produced vasoconstriction with different intensity. These experiments were carried out before the cloning of the TRPV1 receptor [3]. The blood auto perfusion of the hindlimb made it possible to analyse the microcirculatory effects of capsaicin by laser-Doppler technique from the striated muscle and from the glabrous skin. Capsaicin administered in this way produced vasodilation in the skin meanwhile a strong vasoconstriction was observed in the striated muscle and the perfusion pressure increased. Resiniferatoxin the ultrapotent analogue of capsaicin, at a dose of $1 \mu \mathrm{g} / \mathrm{kg}$ evoked the same effect in a more pronounced fashion and induced a long-term functional irresponsiveness for further capsaicin administration [13].

Taking together the results of experiments we can postulate on the basis of observed blood pressure changes that

A. Phase I is a TRPV1 mediated reflex (Bezold-Jarisch reflex pulmonary chemoreflex)

B. Phase II is TRPV1 mediated with non-neural location presumably smooth muscle expressed receptors sensitive to acute resiniferatoxin treatment, but here we should consider the possibility of endothelin action produced by the involved endothelial cells.

C. Phase III is mediated rather by activation of CB1 than TRPV1 receptors; we can consider it TRPV1 independent.

\section{Baroreflex Sensitivity (BRS)}

Experimental data show, that TRPV1 receptors are located in the aortic arch, ascending aorta and in the carotid sinus [33] and they can be activated by vanilloids and may function as stretch receptors $[34,35]$. The afferents from these receptor sites correspond to $\mathrm{A} \delta$ and $\mathrm{C}$ fibres and they express TRPV1 receptors. Moreover, these sensory afferents release glutamate from the cells of Nucleus Tractus Solitarii (NTS) in the vasomotor centre of the brainstem [36]. RTX pre-treatment deteriorate the BRS especially over the range of $120-130 \mathrm{mmHg}$. Administration of iodo-RTX (a potent TRPV1 antagonist) at a dose of $1 \mu \mathrm{mol} / \mathrm{kg}$ i.e., $754 \mu \mathrm{g} /$ kg i.v., before the baroreflex testing with Phenylephrine (PHE) and Sodium Nitroprusside (SNP) shifted the baroreflex function curves upwards in the higher blood pressure range in accordance with the RTX pre-treatment. Nitric oxide (NO) of sensory neuron or endothelial origin is able to inhibit the carotid sinus BRS with unknown mechanism [37], while prostacyclin of endothelial origin excites the receptive carotid regions and increases baroreceptor activity over a wide range of pressures in normo-and hypertensive rabbits [38].

Hydrogen-Sulfide $\left(\mathrm{H}_{2} \mathrm{~S}\right)$ is discovered as the third gasotransmitter besides Nitric Oxide (NO) and carbon monoxide (CO) [39]. TRPV1 antagonist capsazepine reduced the $\mathrm{H}_{2} \mathrm{~S}$ facilitated carotid BRS in rats [33]. CHO cells expressing cloned TRPV1 receptors did not show any $\mathrm{Ca}^{2+}$ uptake challenged with $\mathrm{NaHS}$ or $\mathrm{Na}_{2} \mathrm{~S}$ [40]. Sympathetic activity enhancement can lead to the decrease of BRS. It turned out recently, that the western diet can induce not only higher fasting glucose, insulin and leptin levels and increased sympathetic nervous system activity, but an increase in mean arterial pressure, which proved to be more serious in TRPV-/mice at the night time, but not in daytime [41]. Thus, the existence of functional TRPV1 receptors is essential for normal BRS functions. Moreover, adipose tissue TNF- $\alpha$ levels are higher in TRPV1-/- mice with western diet intake compared to the wild-type western diet fed animals and to the controls suggesting a role of TRPV1 receptors in leptin release from adipose tissue [41]. Detailed investigations are needed to further characterize the TRPV1 receptor function in the BRS mechanisms.

TRPV1 expressing sensory neurons innervating the heart are playing a role in Cardiac Sympathetic Afferent Reflex (CSAR). The activation of this reflex increases blood pressure and heart rate. Long-term functional unresponsiveness induced by subcutaneous RTX pre-treatment swine led to the loss of primary sensory neurons innervating the heart and projecting to the stellate ganglion and to the dorsal spinal cord. Without these nociceptive afferents CSAR 
was disinhibited and resulted in an elevation in blood pressure [42].

\section{Sensory Neuron Dependent Vasodilation}

TRPV1 excitation induce the release of sensory neuropeptides from sensory neurons, such as CGRP and SP and these neuropeptides will cause vasorelaxation in arterioles, thus the sensory nerves, especially $\mathrm{A} \delta$ and $\mathrm{C}$ fibres have dual sensory-efferent functions [43]. Perivascular nerves in the skin and the dura mater were shown to express TRPV1 receptors and to contain CGRP and SP, and have role in migraine pathophysiology [44]. The capsaicin-induced arteriolar vasodilation mediated by the innervating sensory neurons do not need any of axonal conduction. The accompanying neurogenic inflammation showed no sensitivity to previously administered $1 \%$ solution of procaine in the dorsal skin of the rat paw [45]. Electrically evoked (20V, $0.5 \mathrm{~ms}, 2-5 \mathrm{~Hz}, 5 \mathrm{~min})$ antidromic excitation of dorsal roots capable to induce vasodilation in the skin and inner organs of the rat measured by Evans blue plasma extravasation [46] and antidromic excitation $(10 \mathrm{~V}, 0.1-1.0 \mathrm{~ms}, 2 \mathrm{~Hz}, 15 \mathrm{~s})$ of saphenous nerve of the rat lead to flow increase in the skin measured by LaserDoppler flowmetry [47].

Dux and her co-workers reported trigeminal nerve origin capsaicin-sensitive sensory nerve mediated vasodilation in the dura mater of the rat when capsaicin was applied topically at the concentration of $50-100 \mathrm{nM}$, although blood flow decrease (i.e. vasoconstriction) was observed at micromolar concentration range $(1$ and $10 \mu \mathrm{M})$ [48]. These experiments suggest that multiple (at least two) mechanisms can play a role in the regulation and the sensitivity of them to capsaicin is dose-dependent. Dural vasodilation was observed during supramaximal electrical stimulation of the trigeminal ganglion, but it was attributed to capsaicin-insensitive sensory nerves [49], which is in controversy with formerly discussed observation [48]. The question arises, what can be the physiological role of such a system over the role of local regulation? The cell bodies of primary sensory neurons are located in the dorsal root ganglia and as a pseudo unipolar neuron, the axon after leaving the cell body splits into two branches, one extends to the central nervous system and arrives into the dorsal horn, mostly Rexed's lamina II of the spinal cord while the other end extends to the periphery and innervates skin, joints, muscles and obviously inner organs (involving other Rexed's laminae as a relay) [46]. So, in this system, synapses cannot restrict the direction of electrical propagation, thus theoretically "physiological antidromic" excitation can arrive from the central nervous system to the periphery using this possible way of propagation. To test this possibility neuronal electrical activity was recorded from the peripheral end of the spinal dorsal root's central stump. These neurons were generally silent but a massive firing was observed in various pathophysiological states such as hypoxia and hypercapnia
[50]. The relevance of this mechanism is undefined, but the loss of these sensory neurons excludes the possibility of local and remote vascular calibre regulation.

Capsaicin administration $(0.1-7.0 \mu \mathrm{g} / \mathrm{kg})$ into the superior mesenteric artery of the dog in situ caused dose-dependent blood flow increase measured by an electromagnetic flow-probe in the trunk of the superior mesenteric artery. The authors mention in a sentence that higher doses of capsaicin $(10-160 \mu \mathrm{g} / \mathrm{kg})$ injected into the same artery could evoke a pronounced decrease in intestinal blood flow [51]. The well-known cardiotoxic Adriamycin was able to reduce the capsaicin-induced cutaneous neurogenic sensory vasodilatation and CGRP release in rats, which might have an importance and can be indicative of the imminent cardiotoxicity of the drug in clinical situations [52]. An interesting recent observation that TRPV1 excitation by high millimolar concentration of phenylephrine accompanying CGRP release and meningeal vasodilation [53], but the relevance and importance of this finding has not been determined yet. Numerous experiments performed on isolated rat aortic ring preparations showed the relaxing effect of capsaicin and investigated the effects of metabolic syndrome induced by high fat diet feeding [54]. Long-term alimentary capsaicin intake showed an improvement of aortic ring relaxations in high fat diet-induced vascular dysfunction.

The relevance of these kind of experiments is questionable, because the aorta is minimally responsible for the maintenance of high blood pressure, but the peripheral arterioles especially those supplying skeletal muscles are highly involved. Capsaicininduced relaxation of isolated pressurized mice coronary micro vessels was inhibited by SB366791 (TRPV1 antagonist), L-NAME (NOS inhibitor), iberiotoxin and Penetrim A (large conductance calcium sensitive potassium channel (BK) inhibitor) indicating the involvement of TRPV1, eNOS and BK channels. Marked attenuation was observed in myocardial blood flow in genetically diabetic mice. Endothelial denudation (passing through an air bubble in the lumen of the vessel) could inhibit the vasodilatatory effect of capsaicin. Moreover, the TRPV1 expression was reduced in the hearts of diabetic $\mathrm{db} / \mathrm{db}$ mice [55]. It is worthy to mention that propofol-induced coronary relaxation was not influenced in TRPV1 KO but only in double knock out (TRPAV-/-) mice [56].

Oxidative stress involves the production of Reactive Oxygen Species (ROS), which can lead to lipid peroxidation, peroxidation of $\omega-6$ or $\omega-3$ polyunsaturated fatty acids in cells producing $\alpha, \beta$ unsaturated hydroxyalkenal products such as 4-hydroxy-2-nonenal (4-HNE), 4-hydroxy-2-hexenal (4-HHE), 4-oxynonenal (4-ONE). The normal low level $(0.5-5.0 \mu \mathrm{M})$ of 4-HNE product in cells is required to maintain physiological cell functions (normal proliferation, differentiation, antioxidant defense), whereas high concentrations

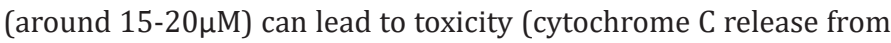


mitochondria, caspase enzyme induction and other toxic effects), and to cell death. Elevated levels of 4-HNE was observed in diabetes and atherosclerosis and it seemed to be crucial to elucidate its role in TRPV1-mediated vascular signalling [57]. Oroszi et al. [58] reported that capsaicin (10-7 M for $5 \mathrm{~min}$ ) can induce an increase in coronary flow and heart rate in Langendorff-perfused guinea-pig hearts, which was shown to be mediated by endogenous NO and CGRP production but the source of these mediators was not verified. TRPV1 channels can be sensitized by injury or inflammation playing a role in pain sensation and concomitant vasodilation [59], but it turned out that TRPV1 receptors play a minor role in acute noxious heat detection [60].

\section{The Possible Role of TRPV1 Expression in Endothe- lial Cells}

It has been demonstrated that endothelial cells derived from human brain capillaries and micro vessels (HBEC) can express TRPV1 receptors with coexpression of endocannabinoid receptors [61] and even the endocannabinoid 2-arachidonoyl-glycerol (2-AG) can activate the TRPV1 receptors. Moreover, it has been described that TRPV1 activation increased the expression of protein kinase A/uncoupling protein 2 (PKA/UCP2) in endothelial cells, improving mitochondrial function deteriorated by high fat containing diet and thus reduced coronary artery lesions [62]. Capsaicin rich food could reduce the endothelial oxidative stress in diabetic mice by TRPV1 mediated UCP2 upregulation [63]. The development of endothelial dysfunction can be considered as a prerequisite of atherosclerotic plaque formation, thus maintaining or improving the endothelial function can be crucial in prevention of cardiovascular diseases and hypertension development $[12,64]$.

A 4-minute infusion of capsaicin in the dose of $10,20,100 \mu \mathrm{g} /$ $\mathrm{kg}$ intravenously into mice in vivo could increase myocardial blood flow measured by Myocardial Contrast Echocardiography (MCE). This flow increase was blocked by capsazepine, L-NAME and was not seen in TRPV1 KO mice. It is interesting, that the flow increase evoked by $100 \mu \mathrm{g} / \mathrm{kg}$ capsaicin was 1.5 -fold less than that of $20 \mu \mathrm{g} /$ $\mathrm{kg}$ capsaicin administration though it proved to be significant compared to the control value [55]. This capsaicin mediated relaxation was attributed to endothelial-dependent NO production, because L-NAME treatment or endotheliallayer disruption abolished the effect. TRPV1 expression was described in rat coronary artery endothelial cells functionally playing role in $\mathrm{Ca}^{2+}$-dependent $\mathrm{NO}$ release [65]. The activation of TRPV1 channels in endothelial cells increases $\mathrm{Ca}^{2+}$ influx increasing the phosphorylation of eNOS, thus increasing its activity and producing a higher level of NO (Figure 3 in review of Randhawa \& Jaggi) [66]. The increased level of NO in the endothelial cells can inhibit the translation of ProET-1 and also can inhibit the Ang-II and thrombin regulated release of transGolgi vesicles containing premature and matured ET-1 products
[67]. These experiments let us tend to conceive that endothelial TRPV1 receptor excitation can lead to more vasodilation instead of vasoconstriction. However, endothelin in endothelial cells were identified several decades ago and other laboratories showed the functional role of endothelin released from endothelial cell layers (detailed in the next section).

\section{Experiments on the Analysis of Capsaicin-Induced Vasoconstriction}

It was known from the mid of 50s of last century from in vivo experiments, that in spinalized cats treated with ganglion-blocking agents or after treatment with $\alpha$-adrenoceptor blocker, the rise in blood pressure evoked by intravenous injection of capsaicin was not reduced $[25,68,69]$ and, therefore, it was attributed to a "direct" vascular effect of capsaicin. Several other authors strengthened this view by isolated arterial ring experiments in vitro discussed by Czikora [70], but it remained without interest for a long time. Species differences and sensitivity of arteries from different part of the body were described by Duckles [71] in 1986. Cat middle cerebral arteries responded with vascular smooth muscle contraction to capsaicin challenge, while guinea pig thoracic aorta and carotid artery showed smooth muscle relaxation. The relaxation was sensitive and could be eliminated by high dose of subcutaneous capsaicin (in two doses all in all $350 \mathrm{mg} / \mathrm{kg}$ ) treatment in vivo prior to the in vitro experiments.

Endothelium removal did not influence the capsaicin-induced vasoconstriction in the cat middle cerebral artery. Duckles concluded that vasoconstriction is a direct smooth muscle effect and the observed vasodilation is due to the release of an "unidentified bioactive substance distinct from substance P" [71]. The classical artery ring technique was used further to analyse the capsaicininduced vasoconstriction. The drawback of this technique is that only large arteries can be investigated, and it turned out that some of these large arteries of different species are completely unresponsive to capsaicin. We tested rat aorta, rabbit auricular artery, swine mesenteric arteries, but none of them responded to capsaicin challenge (unpublished observations). One can guess that the TRPV1 receptor expression is not ubiquiter for all arteries of different calibre and large species differences can be observed, too. Finally, we found canine mesenteric arteries for investigation [14]. In these experiments the mesenteric arteries obtained from dogs were placed into a refrigerator maintained at $4^{\circ} \mathrm{C}$ in Krebs solution for one or two weeks with the intention to get rid of the periarterial nerves [72]. Arteries after this cold-storage produced significantly more intensive vasoconstriction challenged by capsaicin, although the mechanism was not elucidated.

Pressurized skeletal muscle arterioles of the gracilis muscle of the rat and canine coronary arteries were used to characterize the smooth muscle expressed TRPV1 receptors by some agonists 
such as capsaicin, MSK-195, JYL-79, JYL-1511, JYL-273 and resiniferatoxin. Capsaicin produced reproducible contractions of the arteries, but surprisingly, the ultrapotent RTX was unable to excite these receptors and no vascular response was observed, however long-term functional unresponsiveness developed and capsaicin completely lost the effect [70]. Here again should be stressed, that differences between species and vascular beds may vary significantly. Hence, Ossabaw miniature swine right coronary arteries responded to capsaicin challenge with vasorelaxation in in vitro isometric tension experiments which proved to be endothelium dependent and could be inhibited by capsazepine [73].

In another series of experiments isolated working rathearts were used, but capsaicin in the concentration of $10^{-9}-10^{-6} \mathrm{M}$ induced dose dependent decrease in Heart Rate (HR), Aortic Flow (AF), Coronary Flow (CF) and Left Ventricular Developed Pressure (LVDP). The magnitude of the response evoked by $10^{-8} \mathrm{M}$ capsaicin proved to be around the same evoked by $10^{-10} \mathrm{M}$ endothelin administration. The non-selective endothelin receptor blocking substance PD142893 was able to reduce the effects of capsaicin and endothelin in the same manner. In this series of experiments the source of endothelin was not investigated but the state of art in that time excluded the existence of TRPV1 receptor expression in endothelial cells or cardiac myocytes. Endothelin was concluded to be released from sensory nerve terminals as a sensory neuropeptide [74]. Two years later the release of endothelin of neural origin induced by capsaicin administration in the same experimental setup was measured by radioimmunoassay in the same laboratory.

Endothelin release proved to be dependent of extracellular $\mathrm{Ca}^{2+}$ concentration. Twofold and sevenfold increase was observed in endothelin release after the administration of $10^{-8}$ and $10^{-7} \mathrm{M}$ capsaicin, respectively. The source of endothelin from endothelial cells was excluded because treatment with Triton X-100 (considered as an endothelial cell destructive agent) remained without effect on capsaicin-induced vasoconstriction of coronary arteries [75]. Ohanyan et al. [76] reported endothelin receptor (ETA) receptor mediated vasoconstriction evoked by capsaicin infusion in mice in vivo experiments. The administration of FR139317 as an $\mathrm{ET}_{\mathrm{A}}$ receptor antagonist could abolish the vasoconstrictive effect of capsaicin [76]. Moreover, this capsaicin-induced vasoconstriction was blunted in diabetic $\mathrm{db} / \mathrm{db}$ mice. They found that incubation fluid containing capsaicin could induce the release of endothelin from cultured endothelial cells in vitro. Endothelin-1 is of an endothelial cell origin 21-amino acid containing peptide cleaved from big-endothelin-1 by certain enzymes (endothelin converting enzyme, neprilysin) [67], and endothelin-1 caused mechanical allodynia that was sensitive to SB-366791 TRPV1 antagonist [77]. Vasoconstriction can be seen not only in the territory of skeletal muscles and in the arterial supply of the gut, but it was described in a sensory organ such as cochlea. Capsaicin infusion at a dose of $150 \mathrm{nM} / \mathrm{min}$ into the anterior inferior cerebellar artery an immediate blood flow decrease was observed in the cochlear blood flow measured by laser Doppler flowmetry in Guinea pigs [78].

Pulmonary arteries and arterioles are of a special importance in the development of Idiopathic Pulmonary Arterial Hypertension (IPAH), thus the role of expressed TRPV1 receptors in this smooth muscle cells should be clarified. Cytoskeletal reorganization of pulmonary artery smooth muscle cells (PASMC) proved to be TRPV1 dependent during hypoxia-induced cell migration [79]. Song et al. [80] reported that human PASMCs from IPAH patients occurred to be around 8-times more sensitive to capsaicin challenge characterized by intracellular $\mathrm{Ca}^{2+}$ increase compared to the normal human PASMC samples (IPAH PASMC capsaicin sensitivity: $275 \mu \mathrm{M}$ vs. normal PASMC sensitivity: $35 \mu \mathrm{M}$ ). The phosphorylated CREB (cAMP response element-binding protein) intracellular level proved to be higher in IPAH PASMCs as well. Iodo-RTX treatment completely abolished, capsazepine dose-dependently reduced the capsaicin-mediated increase in intracellular $\mathrm{Ca}^{2+}$ concentration of IPAH PASMC samples, while TRPV1 mRNA and protein expression levels were increased. From pathophysiological point of view, it is important to note, that IPAH PASMC proliferation was inhibited by TRPV1 antagonists [80]. Over the direct PASMC effects of TRPV1 agonists, the anti-inflammatory sensory neuropeptide release (somatostatin, PACAP) from primary sensory neurons innervating the lungs should be considered as a modulator of arteriolar vasomotion [81].

\section{Vascular Locations of TRPV1 Receptors Presented by Reporter Mice}

A molecular biology technique started to serve the discoveries in TRPV1 receptor pharmacology recently. Reporter genes in plasmid form can be inserted into the mouse genome for example gfp and lacZ producing green fluorescein protein and $\beta$-galactosidase, respectively and these products can be detected by fluorescent microscopy and with the determination of enzyme activity. Validated TRPV1-related reporter mice were described recently. TRPV1 ${ }^{\text {PLAP- }}$ nlacz operates with human Placental Alkaline Phosphatase (PLAP) and Nuclear $\beta$-galactosidase (nLacZ) controlled by the originally existing TRPV1 promoter in the mouse genome [82]. Another model was established with BAC transgenic mice 10 expressing tdTomato under the control of TRPV1-Cre(Tg(Trpv1-cre)1Hoon, (developed by Mark A. Hoon) promoter for detection of existing TRPV1 expressions in sensory neurones [83]. Reporter mice were applied to prove the functional existence of TRPV1 receptors in Arteriolar Smooth Muscle (ASM) cells in thermoregulatory organs such as skin, tongue, trachea and cremaster muscle [82]. 
For the first time sex difference was observed with female predominance in arteriolar TRPV1 receptor expression in urinary bladder using TRPV1-Cre:td Tomato mice [84]. It is worthy to mention that capillaries do not express TRPV1 [84], which is obvious if we consider the fact, that the wall of capillaries do not contain smooth muscle cells. These experiments disclose the possibility of endothelial TRPV1 expression in endothelial cells of the capillary bed and makes more complex the functional role of TRPV1 receptors in endothelial cells of different vascular beds. Moreover, an age-related increase in the expression of TRPV1 in ASM cells was described and it should be emphasized that young animals (less than 28days of age) do not express TRPV1 receptors at all. This age-related change in expression pattern is completely different from that experienced with TRPV1 receptors located on sensory neurons. Functionally these receptors mediate vasoconstriction, if they are excited by capsaicin and this effect was inhibited by the TRPV1 receptor antagonist BCTC. The physiological role of this receptors in the urinary bladder is unclear, but considering the possibility of inflammation accompanying cystitis (more frequently observed in female human beings) the excitation of these receptors can lead to haemorrhage development.

\section{Conclusion and Perspectives}

The pharmacological exploitation of TRPV1 receptor antagonists in the treatment of chronic pain has not been successful yet. All of the developed compounds failed during the clinical studies because of the development of unwanted effects such as hyperthermia. TRPV1 receptors localized not only on primary sensory neurons, but on vascular smooth muscle cells and even participate in the chemo- and in the baroreflex. Figure 1 summarises the physiological processes where TRPV1 receptors can play a role in the homeostasis of vascular system. As the mechanism of essential hypertension's development is not exactly known, thus further investigations are needed to clarify the role of TRPV1 receptor in the fine tuning of baroreflex and targeted experiments should be carried out how we can restore the normal blood pressure exploiting TRPV1 receptors in this system.

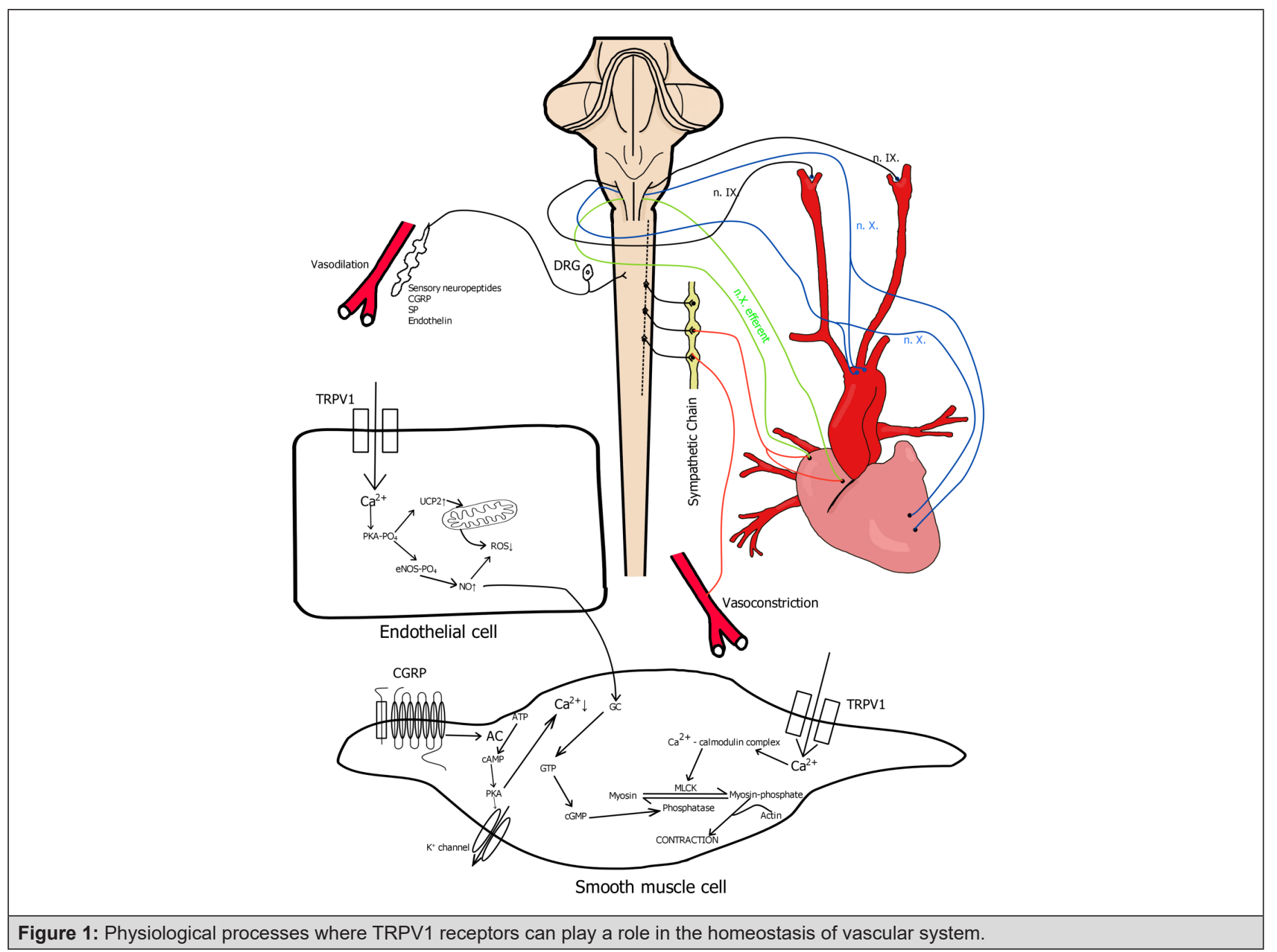


The former experiments using antibodies to visualize TRPV1 receptors should be reconsidered from histochemical point of view because of the experienced unspecific binding. Mapping of the receptor in different parts of the body will be complete and more reliable by using the Reporter Mice technique. Unfortunately, this technique is restricted to mice, which underlines the importance of functional tests in decision making process about the relevance. The experiments using whole living (anaesthetized, conscious) animals should be not neglected only because of its complexity and even reliable translational medicine models are a need of sore to develop just to predict the upcoming drug candidates' successfulness in clinical studies.

\section{References}

1. Gaudet R (2008) TRP channels entering the structural era. The Journal of physiology 586(15): 3565-3575.

2. Yuan P (2019) Structural biology of thermo TRPV channels. Cell calcium 84: 102106.

3. Caterina MJ, Schumacher MA, Tominaga M, Rosen TA, Levine JD, et al. (1997) The capsaicin receptor: a heat-activated ion channel in the pain pathway. Nature 389(6653): 816-824.

4. Szolcsanyi J, Sandor Z (2012) Multisteric TRPV1 nocisensor: a target for analgesics. Trends in pharmacological sciences 33(12): 646-655.

5. Nilius B, Szallasi A (2014) Transient receptor potential channels as drug targets: from the science of basic research to the art of medicine. Pharmacological reviews 66(3): 676-814.

6. Gouin O, L'Herondelle K, Lebonvallet N, Le Gall Ianotto C, Sakka M, et al. (2017) TRPV1 and TRPA1 in cutaneous neurogenic and chronic inflammation: pro-inflammatory response induced by their activation and their sensitization. Protein \& cell 8(9): 644-661.

7. Ursu D, Knopp K, Beattie RE, Liu B, Sher E (2010) Pungency of TRPV1 agonists is directly correlated with kinetics of receptor activation and lipophilicity. European journal of pharmacology 641(2-3): 114-122.

8. Kuzma M, Fodor K, Almasi A, Mozsik G, Past T, et al. (2019) Toxicokinetic Study of a Gastroprotective Dose of Capsaicin by HPLC-FLD Method. Molecules 24(15): 2848

9. Nolden AA, Lenart G, Hayes JE (2019) Putting out the fire - Efficacy of common beverages in reducing oral burn from capsaicin. Physiology \& behaviour 208: 112557.

10. Molinas AJR, Desmoulins LD, Hamling BV, Butcher SM, Anwar IJ, et al. (2019) Interaction between TRPV1-expressing neurons in the hypothalamus. Journal of neurophysiology 121(1): 140-151.

11. Bodo E, Biro T, Telek A, Czifra G, Griger Z, et al. (2005) A hot new twist to hair biology: involvement of vanilloid receptor-1 (VR1/TRPV1) signalling in human hair growth control. The American journal of pathology 166(4): 985-998.

12. Yang D, Luo Z, Ma S, Wong WT, Ma L, et al. (2010) Activation of TRPV1 by dietary capsaicin improves endothelium-dependent vasorelaxation and prevents hypertension. Cell metabolism 12(2): 130-141.

13. Kark T, Bagi Z, Lizanecz E, Pasztor ET, Erdei N, et al. (2008) Tissuespecific regulation of microvascular diameter: opposite functional roles of neuronal and smooth muscle located vanilloid receptor-1. Molecular pharmacology 73(5): 1405-1412.

14. Porszasz R, Porkolab A, Ferencz A, Pataki T, Szilvassy Z, et al. (2002) Capsaicin induced nonneural vasoconstriction in canine mesenteric arteries. European journal of pharmacology 441(3): 173-175
15. Bertin S, Aoki Nonaka Y, de Jong PR, Nohara LL, Xu H, et al. (2014) The ion channel TRPV1 regulates the activation and proinflammatory properties of $\mathrm{CD}^{4+} \mathrm{T}$ cells. Nature immunology 15(11): 1055-1063.

16. Li L, Chen J, Ni Y, Feng X, Zhao Z, et al. (2012) TRPV1 activation prevents non-alcoholic fatty liver through UCP2 upregulation in mice. Pflugers Archiv European journal of physiology 463(5): 727-732.

17. Harper AG, Brownlow SL, Sage SO (2009) A role for TRPV1 in agonistevoked activation of human platelets. Journal of thrombosis and haemostasis: JTH 7(2): 330-338.

18. Toth A, Czikora A, Pasztor ET, Dienes B, Bai P, et al. (2014) Vanilloid receptor-1 (TRPV1) expression and function in the vasculature of the rat. The journal of histochemistry and cytochemistry official journal of the Histochemistry Society 62(2): 129-144.

19. Sand CA, Grant AD, Nandi M (2015) Vascular Expression of Transient Receptor Potential Vanilloid 1 (TRPV1). The journal of histochemistry and cytochemistry official journal of the Histochemistry Society 63(6): 449-453.

20. Schumacher MA, Eilers H (2010) TRPV1 splice variants: structure and function. Frontiers in bioscience 15: 872-882.

21. Malinowska B, Kwolek G, Gothert M (2001) Anandamide and methanandamide induce both vanilloid VR1- and cannabinoid CB1 receptor-mediated changes in heart rate and blood pressure in anaesthetized rats. Naunyn-Schmiedeberg's archives of pharmacology 364(6): 562-569.

22. Jancso G, Such G (1983) Effects of capsaicin applied perineurally to the vagus nerve on cardiovascular and respiratory functions in the cat. The Journal of physiology 341: 359-370.

23. Gu Q, Lin RL, Hu HZ, Zhu MX, Lee LY (2005) 2-aminoethoxydiphenyl borate stimulates pulmonary $\mathrm{C}$ neurons via the activation of TRPV channels. American journal of physiology Lung cellular and molecular physiology 288(5): 932-941.

24. Lin YS, Lee LY (2002) Stimulation of pulmonary vagal C-fibres by anandamide in anaesthetized rats: role of vanilloid type 1 receptors. The Journal of physiology 539: 947-955.

25. Porszasz J, Gyorgy L, Porszasz Gibiszer K (1955) Cardiovascular and respiratory effects of capsaicin. Acta physiologica Academiae Scientiarum Hungaricae 8(1): 61-76.

26. Kossakowski R, Schlicker E, Toczek M, Weresa J, Malinowska B (2019) Cannabidiol Affects the Bezold-Jarisch Reflex via TRPV1 and 5-HT3 Receptors and Has Peripheral Sympathomimetic Effects in Spontaneously Hypertensive and Normotensive Rats. Frontiers in pharmacology 10: 500 .

27. McQueen DS, Bond SM, Smith PJ, Balali Mood K, Smart D (2004) Cannabidiol lacks the vanilloid VR1-mediated vasorespiratory effects of capsaicin and anandamide in anaesthetised rats. European journal of pharmacology 491(2-3): 181-189.

28. Lin RL, Gu Q, Lee LY (2017) Hypersensitivity of Vagal Pulmonary Afferents Induced by Tumor Necrosis Factor Alpha in Mice. Frontiers in physiology 8: 411

29. Morton PE, Hicks A, Ortiz Zapater E, Raghavan S, Pike R, et al. (2016) TNF-alpha promotes CAR-dependent migration of leukocytes across epithelial monolayers. Scientific reports 6: 26321.

30. Colquhoun EQ, Eldershaw TP, Bennett KL, Hall JL, Dora, et al. (1995) Functional and metabolic evidence for two different vanilloid $\left(\mathrm{VN}_{1}\right.$ and $\mathrm{VN}_{2}$ ) receptors in perfused rat hindlimb. Life sciences 57(2): 91-102.

31. Cameron Smith D, Colquhoun EQ Ye JM, Hettiarachchi M, Clark MG (1990) Capsaicin and dihydrocapsaicin stimulate oxygen consumption in the perfused rat hindlimb. International journal of obesity 14(3): 259270 . 
32. Eldershaw TP, Colquhoun EQ, Bennett KL, Dora KA, Clark MG (1994) Resiniferatoxin and piperine: capsaicin-like stimulators of oxygen uptake in the perfused rat hindlimb. Life sciences 55(5): 389-397.

33. Yu W, Liao Y, Huang Y, Chen SY, Sun Y, et al. (2017) Endogenous Hydrogen Sulfide Enhances Carotid Sinus Baroreceptor Sensitivity by Activating the Transient Receptor Potential Cation Channel Subfamily V Member 1 (TRPV1) Channel. Journal of the American Heart Association 6(5).

34. Sun H, Li DP, Chen SR, Hittelman WN, Pan HL (2009) Sensing of blood pressure increase by transient receptor potential vanilloid 1 receptors on baroreceptors. The Journal of pharmacology and experimental therapeutics 331(3): 851-859.

35. Liu C, Montell C (2015) Forcing open TRP channels: Mechanical gating as a unifying activation mechanism. Biochemical and biophysical research communications 460(1): 22-25.

36. Jin YH, Bailey TW, Li BY, Schild JH, Andresen MC (2004) Purinergic and vanilloid receptor activation releases glutamate from separate cranial afferent terminals in nucleus tractus solitarius. The Journal of neuroscience: the official journal of the Society for Neuroscience 24(20): 4709-4717.

37. Matsuda T, Bates JN, Lewis SJ, Abboud FM, Chapleau MW (1995) Modulation of baroreceptor activity by nitric oxide and S-nitrosocysteine. Circulation research 76(3): 426-433.

38. Xie PL, Chapleau MW, McDowell TS, Hajduczok G, Abboud FM (1990) Mechanism of decreased baroreceptor activity in chronic hypertensive rabbits. Role of endogenous prostanoids. The Journal of clinical investigation 86(2): 625-630.

39. Powell CR, Dillon KM, Matson JB (2018) A review of hydrogen sulfide $\left(\mathrm{H}_{2} \mathrm{~S}\right)$ donors: Chemistry and potential therapeutic applications. Biochemical pharmacology 149: 110-123.

40. Hajna Z, Saghy E, Payrits M, Aubdool AA, Szoke E, et al. (2016) CapsaicinSensitive Sensory Nerves Mediate the Cellular and Microvascular Effects of H2S via TRPA1 Receptor Activation and Neuropeptide Release. Journal of molecular neuroscience MN 60(2): 157-170.

41. Zhong B, Ma S, Wang DH (2019) Ablation of TRPV1 Elevates Nocturnal Blood Pressure in Western Diet-fed Mice. Current hypertension reviews 15(2): 144-153.

42. Yoshie K, Rajendran PS, Massoud L, Kwon O, Tadimeti V, et al. (2018) Cardiac vanilloid receptor-1 afferent depletion enhances stellate ganglion neuronal activity and efferent sympathetic response to cardiac stress. American journal of physiology Heart and circulatory physiology 314(5): 954-966.

43. Szolcsanyi J (2004) Forty years in capsaicin research for sensory pharmacology and physiology. Neuropeptides 38(6): 377-384.

44. Del Fiacco M, Quartu M, Boi M, Serra MP, Melis T, et al. (2015) TRPV1, CGRP and SP in scalp arteries of patients suffering from chronic migraine. Journal of neurology neurosurgery and psychiatry 86(4): 393-397.

45. Jancso N, Jancso Gabor A, Szolcsanyi J (1968) The role of sensory nerve endings in neurogenic inflammation induced in human skin and in the eye and paw of the rat. British journal of pharmacology and chemotherapy 33(1): 32-41.

46. Pinter E, Szolcsanyi J (1995) Plasma extravasation in the skin and pelvic organs evoked by antidromic stimulation of the lumbosacral dorsal roots of the rat. Neuroscience 68(2): 603-614.

47. Gamse R, Saria A (1987) Antidromic vasodilatation in the rat hind paw measured by laser Doppler flowmetry: pharmacological modulation. Journal of the autonomic nervous system 19(2): 105-111.

48. Dux M, Santha P, Jancso G (2003) Capsaicin-sensitive neurogenic sensory vasodilatation in the dura mater of the rat. The Journal of physiology 552(3) 859-867.
49. Peitl B, Petho G, Porszasz R, Nemeth J, Szolcsanyi J (1999) Capsaicininsensitive sensory efferent meningeal vasodilatation evoked by electrical stimulation of trigeminal nerve fibres in the rat. British journal of pharmacology 127(2): 457-467.

50. Petho G, Porszasz R, Peitl B, Szolcsanyi J (1999) Spike generation from dorsal roots and cutaneous afferents by hypoxia or hypercapnia in the rat in vivo. Experimental physiology 84(1): 1-15.

51. Rozsa Z, Jancso G, Varro V (1984) Possible involvement of capsaicinsensitive sensory nerves in the regulation of intestinal blood flow in the dog. Naunyn-Schmiedeberg's archives of pharmacology 326(4): 352356.

52. Boros K, Jancso G, Dux M, Fekecs Z, Bencsik P, et al. (2016) Multiple impairments of cutaneous nociceptor function induced by cardiotoxic doses of Adriamycin in the rat. Naunyn-Schmiedeberg's archives of pharmacology 389(9): 1009-1020.

53. Dux M, Babes A, Manchen J, Sertel Nakajima J, Vogler B, et al. (2019) High-dose phenylephrine increases meningeal blood flow through TRPV1 receptor activation and release of calcitonin gene-related peptide. European journal of pain 24(2): 383-397.

54. Zhu ZY, Zhang LL, Wang PJ, Ma LQ Wang LJ, et al. (2011) Activation of transient receptor potential vanilloid 1 inhibits RhoA/Rho kinase and improves vasorelaxation dysfunction mediated by high-fat diet in mice. Zhongguo yi xue ke xue yuan xue bao Acta Academiae Medicinae Sinicae 33(6): 600-605.

55. Guarini G, Ohanyan VA, Kmetz JG, DelloStritto DJ, Thoppil RJ, et al. (2012) Disruption of TRPV1-mediated coupling of coronary blood flow to cardiac metabolism in diabetic mice: role of nitric oxide and BK channels. American journal of physiology Heart and circulatory physiology 303(2): 216-223.

56. Sinharoy P, Bratz IN, Sinha S, Showalter LE, Andrei SR, et al. (2017) TRPA1 and TRPV1 contribute to propofol-mediated antagonism of U46619-induced constriction in murine coronary arteries. PloS one 12(6): 0180106.

57. Dello Stritto DJ, Sinharoy P, Connell PJ, Fahmy JN, Cappelli HC, et al. (2016) 4-Hydroxynonenal dependent alteration of TRPV1-mediated coronary microvascular signalling. Free radical biology \& medicine 101: 10-19.

58. Oroszi G, Szilvassy Z, Nemeth J, Tosaki A, Szolcsanyi J (1999) Interplay between nitric oxide and CGRP by capsaicin in isolated guinea-pig heart. Pharmacological research 40(2): 125-128.

59. Carnevale V, Rohacs T (2016) TRPV1: A Target for Rational Drug Design. Pharmaceuticals 9(3): 52 .

60. Yarmolinsky DA, Peng Y, Pogorzala LA, Rutlin M, Hoon MA, et al. (2016) Coding and Plasticity in the Mammalian Thermosensory System. Neuron 92(5): 1079-1092.

61. Golech SA, McCarron RM, Chen Y, Bembry J, Lenz F, et al. (2004) Human brain endothelium: coexpression and function of vanilloid and endocannabinoid receptors. Brain research Molecular brain research 132(1): 87-92.

62.Xiong S, Wang P, Ma L, Gao P, Gong L, et al. (2016) Ameliorating Endothelial Mitochondrial Dysfunction Restores Coronary Function via Transient Receptor Potential Vanilloid 1-Mediated Protein Kinase A/ Uncoupling Protein 2 Pathway. Hypertension 67(2): 451-460.

63. Sun J, Pu Y, Wang P, Chen S, Zhao Y, et al. (2013) TRPV1-mediated UCP2 upregulation ameliorates hyperglycaemia-induced endothelial dysfunction. Cardiovascular diabetology 12: 69.

64. Ma L, Zhong J, Zhao Z, Luo Z, Ma S, et al. (2011) Activation of TRPV1 reduces vascular lipid accumulation and attenuates atherosclerosis. Cardiovascular research 92(3): 504-513. 
65. Torres Narvaez JC, Mondragon Ldel V, Varela Lopez E, Perez Torres I, Diaz Juarez JA, et al. (2012) Role of the transient receptor potential vanilloid type 1 receptor and stretch-activated ion channels in nitric oxide release from endothelial cells of the aorta and heart in rats. Experimental and clinical cardiology 17(3): 89-94.

66. Randhawa PK, Jaggi AS (2018) A Review on Potential Involvement of TRPV1 Channels in Ischemia-Reperfusion Injury. Journal of cardiovascular pharmacology and therapeutics 23(1): 38-45.

67. Houde M, Desbiens L, D Orleans Juste P (2016) Endothelin-1: Biosynthesis, Signalling and Vasoreactivity. Advances in pharmacology 77: 143-175.

68. Toda N, Usui H, Nishino N, Fujiwara M (1972) Cardiovascular effects of capsaicin in dogs and rabbits. The Journal of pharmacology and experimental therapeutics 181(3): 512-521.

69. Porszasz J, Such G, Porszaszgibiszer K (1957) Circulatory and respiratory chemoreflexes. I. Analysis of the site of action and receptor types of capsaicine. Acta physiologica Academiae Scientiarum Hungaricae 12(13): 189-205.

70. Czikora A, Lizanecz E, Bako P, Rutkai I, Ruzsnavszky F, et al. (2012) Structure-activity relationships of vanilloid receptor agonists for arteriolar TRPV1. British journal of pharmacology 165(6): 1801-1812.

71. Duckles SP (1986) Effects of capsaicin on vascular smooth muscle. Naunyn-Schmiedeberg's archives of pharmacology 333(1): 59-64.

72. Ito T, Chiba S (1985) Effects of prolonged cold storage on the responsiveness of isolated and perfused canine intermediate auricular artery. Archives internationales de pharmacodynamie et de therapie 275(1): 13-21.

73. Bratz IN, Dick GM, Tune JD, Edwards JM, Neeb ZP, et al. (2008) Impaired capsaicin-induced relaxation of coronary arteries in a porcine model of the metabolic syndrome. American journal of physiology. Heart and circulatory physiology 294(6): 2489-2496

74. Szolcsanyi J, Oroszi G, Nemeth J, Szilvassy Z, Tosaki A (1999) Endothelin release by capsaicin in isolated working rat heart. European journal of pharmacology 376(3): 247-250.

75. Szolcsanyi J, Oroszi G, Nemeth J, Szilvassy Z, Blasig IE, et al. (2001) Functional and biochemical evidence for capsaicin-induced neural endothelin release in isolated working rat heart. European journal of pharmacology 419(2-3): 215-221.
76. Ohanyan VA, Guarini G, Thodeti CK, Talasila PK, Raman P, et al. (2011) Endothelin-mediated in vivo pressor responses following TRPV1 activation. American journal of physiology Heart and circulatory physiology 301(3): 1135-1142.

77. Nodai T, Hitomi S, Ono K, Masaki C, Harano N, et al. (2018) Endothelin-1 Elicits TRP Mediated Pain in an Acid-Induced Oral Ulcer Model. Journal of dental research 97(8): 901-908.

78. Vass Z, Bari F, Jancso G (1994) Possible involvement of capsaicinsensitive sensory nerves in the regulation of cochlear blood flow in the guinea pig. Acta otolaryngologica 114(2): 156-161.

79. Parpaite T, Cardouat G, Mauroux M, Gillibert Duplantier J, Robillard P, et al. (2016) Effect of hypoxia on TRPV1 and TRPV4 channels in rat pulmonary arterial smooth muscle cells. Pflugers Archiv European journal of physiology 468(1): 111-130.

80. Song S, Ayon RJ, Yamamura A, Yamamura H, Dash S, et al. (2017) Capsaicin-induced $\mathrm{Ca}^{2+}$ signalling is enhanced via upregulatedTRPV1 channels in pulmonary artery smooth muscle cells from patients with idiopathic PAH. American journal of physiology Lung cellular and molecular physiology 312(3): 309-325.

81. Zhang X, Ye L, Huang Y, Ding X, Wang L (2019) The potential role of TRPV1 in pulmonary hypertension: Angel or demon? Channels 13(1): $235-246$.

82. Cavanaugh DJ, Chesler AT, Jackson AC, Sigal YM, Yamanaka H, et al. (2011) Trpv1 reporter mice reveal highly restricted brain distribution and functional expression in arteriolar smooth muscle 17cells. The Journal of neuroscience: the official journal of the Society for Neuroscience 31(13): 5067-5077.

83. Mishra SK, Tisel SM, Orestes P, Bhangoo SK, Hoon MA (2011) TRPV1lineage neurons are required for thermal sensation. The EMBO journal 30(3): 582-593.

84. Phan TX, Ton HT, Chen Y, Basha ME, Ahern GP (2016) Sex-dependent expression ofTRPV1 in bladder arterioles. American journal of physiology Renal physiology 311(5): 1063-1073. 